instance, gives an occasional quivering motion to one or both wings which is clearly perceptible to the unaided eye, although propulsion and change of position relatively to air currents seem to be accomplished by strokes of the wings resembling sculling strokes.

It is not the birds, but certain insects, which exhibit quiverings of the wing imperceptible to the eye. The hoverer-fly, Syrphus, for example, can remain in one spot in the air while the wings are vibrating at such a rate as to be invisible, and at the approach of danger, or at will, it may suddenly by some movement, also invisible, transfer itself to a distance of a yard or more, and there continue the wing quiverings, which maintain the body almost stationary.

Is not motion in all flying and swimming things attained by presenting the wings or fins at a suitable angle to the air or water, while at the same time giving a propelling motion to the tail or dorsal fin and body, and also by a sculling motion of the wings or side fins, in the case of some insects and fishes, invisible to the human eye?

Deı by, February 9.

EDWARD D. HEarn.

Demonstration of Peltier and Thomson Effects.

THE following method of demonstrating the Peltier and Thomson effects may be of interest. In Fig. I the current passes through an $\mathrm{S} b-\mathrm{B} i-\mathrm{S} b$ bar, the points of contact being amalgamated to reduce the resistance. Two coils of No. $3^{6}$ covered copper wire are wound on the bismuth

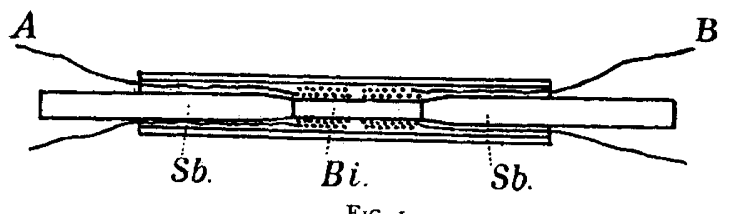

one near each junction, and by means of the leads $\mathrm{A}$ and $\mathrm{B}$ are placed in the gaps of a metre bridge, and a balance produced. On passing a current of $r$ ampere through the bars, one junction is heated and the other cooled, which is indicated by a galvanometer deflection of about $40 \mathrm{~mm}$. due to the change in resistance of the copper coils. The direction indicates a heating where the current flows from $\mathrm{S} b$ to $\mathrm{B} i$, and vice versa.

Fig. 2 shows a similar arrangement for exhibiting the Thomson effect. The bent iron rod is heated to red heat at $C$, and the ends $A$ and $B$ dip into vessels of mercury,

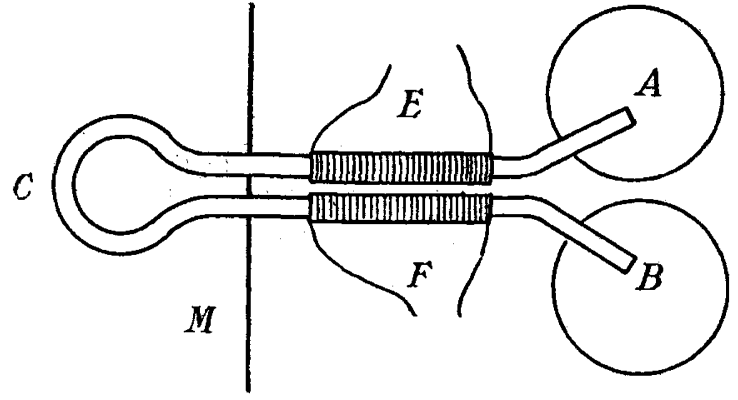

Fig. 2.

thus ensuring a large temperature gradient. On passing a current of to amperes in the direction $\mathrm{ACB}, \mathrm{AC}$ is warmed and $C B$ cooled, showing that the Thomson coefficient is negative. The part EF must be packed in asbestos wool to prevent heating disturbances from outside.

Municipal Technical Institute, Romford Road,

West Ham. E. January 28.

The Formation of Spheres of Liquids.

IN conducting Plateau's experiment for the formation of spheres of liquid in a medium of equal density, it is still eustomary to use oil of some kind in a mixture of alcohol and water. The following method will be found much simpler and more effective. A glass beaker about $10 \mathrm{~cm}$. diameter and $15 \mathrm{~cm}$. high is filled with water at $22^{\circ} \mathrm{C}$. to two-thirds of its height. By means of a pipette, NO. 2 I 55 VOL. 85$]$ roo c.c. of a solution of 30 grams of common salt in I litre of water are discharged at the bottom of the beaker, so as to form a lower layer slightly denser than the water above. A large funnel furnished with a tap, and having a stem I $\mathrm{cm}$. or more in diameter, is now placed centrally in the beaker so that the stem terminates about $7 \mathrm{~cm}$. from the bottom of the vessel. A quantity of commercial orthotoluidine, at a temperature less than $22^{\circ}$, is poured into the funnel, and the tap turned so as to allow the liquid to flow gradually into the water. A sphere of orthotoluidine forms on the end of the stem, the growth of which resembles that of a soap-bubble blown from a pipe.

It is quite easy in this way to make spheres 6 or $8 \mathrm{~cm}$. in diameter, and the red colour of the orthotoluidine renders the procedure visible from a distance. The funnel may be lifted out and the sphere left floating in the water; and on surrounding the beaker by a square glass vessel, also containing water at $22^{\circ}$, the true spherical shape of the drop is seen. If the beaker be surrounded by cold water at $15^{\circ}$, the sphere will elongate in its horizontal diameter and sink, whereas if the surrounding water be at $27^{\circ}$ or more, a vertical elongation will take place, and the sphere will rise and attach itself to the surface of the water in the form of a hanging drop. This behaviour is due to the fact that orthotoluidine and water are equal in density at $22^{\circ}$, but owing to the former liquid possessing a higher coefficient of expansion, it becomes less dense than water above $22^{\circ}$, and more dense at a lower temperature.

It may be added that all the usual experiments with liquid spheres can be carried out in the beaker, and the method of formation has the advantage that a sphere of any desired size may be formed by closing the tap when the requisite quantity of liquid has run out. In the course of a general investigation of liquids which are lighter or denser than water, according to temperature, the writer has found several which may be made to produce spheres at certain temperatures in the manner described, but has found orthotoluidine to be best suited to the experiment.

Chas. R. DARLING.

City and Guilds Technical College, Finsbury, E.C.

\section{Colliery Warnings.}

I HAVE read the letters which have appeared on this subject with considerable interest. We have two theories before us. Bnth theories cunnect the presence of firedamp with changes of atmospheric pressure, but the one considers a time of high pressure as being most likely to cause an outrush of gas, whilst the other regards a falling barometer as the period of greatest danger. It does not seem at all reasonable to suppose that the atmospheric pressure would compress the rock and force out the gas as the Author of the Warnings suggests. Rather would air enter the rock cavities in such circumstances. The tendency for firedamp to escape during a falling barometer would be greater than during a rising barometer, but the evidence only shows a very slight connection to exist between the rise or fall of the barometer and colliery disasters.

The firedamp generated in certain coal measures exists in the rock, apparently, under considerable pressures, and its escape does not appear to be likely to be much affected by atmospheric pressure changes. The Author of the Warnings remarks:-." There was a time when no one guessed that the earth's surface was always on the move. ..." In colliery districts the earth's crust is always on the move, owing to the colliery workings themselves. This movement is not a bodily oscillation-it is an actual rending of the strata for some distance below as well as above the seam being worked. Is it not likely that it is to the formation of fissures in the rock in this way that the gas owes its liberation? Considerable spaces may also be formed by the settling and creep in front of a working face; the firedamp would collect in such spaces and be forced out by further settling. At any rate, it seems clear that the escape of firedamp in quantity is more likely to be the result of some local change rather than to changes of atmospheric pressure.

Inglewood, Longcroft Avenue, Harpenden, R. M. Defley. 